\title{
Damped CAPES 2D Spectral Estimation for Real-Valued Vibration Signals
}

\author{
Danhui Dan, ${ }^{1}$ Jiongxin Gong, ${ }^{1}$ and Yiming Zhao ${ }^{2}$ \\ ${ }^{1}$ Department of Bridge Engineering, Tongji University, Room 711, Bridge Building, 1239 Siping Road, Shanghai 200092, China \\ ${ }^{2}$ Technology Center of Shanghai Construction Group CO., LTD., Shanghai 200431, China \\ Correspondence should be addressed to Danhui Dan; dandanhui@tongji.edu.cn
}

Received 5 March 2014; Revised 19 July 2014; Accepted 22 July 2014; Published 26 August 2014

Academic Editor: Mickaël Lallart

Copyright (c) 2014 Danhui Dan et al. This is an open access article distributed under the Creative Commons Attribution License, which permits unrestricted use, distribution, and reproduction in any medium, provided the original work is properly cited.

\begin{abstract}
We propose a 2D representation in the frequency-decay factor plane of an arbitrary real-world vibration signal. The signal is expressed as the sum of a decayed-attenuation sine term modulated by an amplitude function and a noise residue. We extend the combined approach of Capon estimation and amplitude and phase estimation (CAPES) to damped real vibration signals (DRCAPES). In the proposed DR-CAPES method, the high-resolution amplitude and phase are estimated simultaneously for both angular frequency and decay factor grids. The performance of the proposed approach is tested numerically with noisy vibration data. Results show that the DR-CAPES method has an excellent frequency resolution, which helps to overcome difficulties in spectrum estimation when vibration modes are very close, and a small bias, which makes it suitable for obtaining accurate amplitude spectrums. The results also indicate that the proposed method can accurately estimate the amplitude spectrum with the use of averaging and denoising processes.
\end{abstract}

\section{Introduction}

The normal functioning of engineering structures in dynamic environments is often affected by large and frequent vibrations. These further induce negative effects in terms of comfort in use, fatigue durability, dynamic instability, and safety. Therefore, the structural vibration levels must be assessed under working load conditions and environmental excitation. Typically, these assessments should be conducted in the frequency domain, and thus, structural dynamics experiments have focused on spectral analysis and identification of frequency-domain dynamic characteristics, specifically the structural vibration signal. The vibration level of the structural response is usually described using the Fourier power spectrum $[1,2]$; however, the signal quality could be poor because of noise pollution, short signal length, or inappropriate sampling frequency. Because the Fourier power spectrum frequency often has low resolution and the spectral value estimates are inaccurate, a more suitable and accurate spectral estimation algorithm is needed. The power density spectrum cannot be directly used to assess the vibration amplitude of a certain vibration response. In some cases, the amplitude spectrum is even more important than the power spectrum [3]. Vibrations in the physical world are damped signals and the level of vibration is closely related to the damping. Damping describes the dynamic characteristics and structural performance of the structure; therefore, it must be precisely measured. At present, high-quality damping estimation is very difficult when using the real response signal. In this paper, we propose a nonparametric highprecision spectral estimation algorithm that determines the vibration signal characteristics of engineering structures in order to analyze data from structural dynamics experiments.

One classic category of spectral estimation algorithms is the set of nonparametric methods based on the fast Fourier transform (FFT), in which the frequency resolution is low. However, the FFT has wide applicability, because it has no prespectral shape [3-5]. There is also a category of highresolution parametric methods that require the observed signal to be composed of limited number of superposed sinusoidal signals contaminated by white noise. This is difficult to satisfy in structural vibration signal spectral analyses; therefore, these methods often perform poorly [6]. A third category is the set of nonparametric adaptive filter-bank 
methods with high resolution capability, including the Capon algorithm [7] and the recently proposed amplitude and phase estimation spectral (APES) algorithm [8,9], as well as variants and hybrids of these techniques. These methods have been used successfully for almost twenty years. They make no assumptions about the spectral structure and do not depend on the Fourier transform algorithm; as a result, they can be used for high-resolution spectrum estimation for any stationary signals.

The Capon algorithm performs frequency resolution with greater efficiency than other algorithms, and the APES algorithm estimates spectral values $[10,11]$ with greater accuracy than most of the other algorithms. In 2000, Jakobsson and Stoica combined the two to give the CAPES algorithm, which improves the resolution and estimation accuracy of spectral values [12]. The CAPES algorithm has been used for oneand two-dimensional complex-signal spectral estimation in medical imaging and remote sensing [13-15], and Jakobsson et al. improved the algorithm to handle real one-dimensional vibration signals without damping [16]. In 2001, Stoica and Sundin proposed new versions of the Capon and APES algorithms that can deal with complex one-dimensional damping modulation signals, whose amplitude decays over time [14]. Recently, the researchers focused on the applications and fast computation algorithms of APES and CAPON method [17, 18]. However, when their algorithms are used to estimate the spectrum of the structural vibration directly the accuracy will be worse due to the imaginary part of the signals. Actually, the structural vibration signal is a real one-dimensional damping modulation signal.

Based on existing nonparametric estimation algorithms, we propose a two-dimensional spectral estimation algorithm to transform arbitrary real one-dimensional damping vibration signals in the time-domain into a two-dimensional signal in a joint domain of circular frequency and damping factor. The proposed method is expected to provide a highresolution frequency, high-precision amplitude spectrum, and high-precision damping factor estimations of the vibration signal. Through numerical examples, we verify the effectiveness of the proposed algorithm.

\section{Theory}

2.1. Representation of a Vibration Signal in a $2 D$ Plane. In an $N$-degrees-of-freedom (DOF) vibration system, the response to a steady forced vibration can be written as follows:

$$
x=\sum_{j=1}^{N} x_{j}=\sum_{j=1}^{N} A\left(\omega_{j}, \sigma_{j}\right) e^{-\sigma_{j} t} \sin \left(\omega_{j} t+\varphi\left(\omega_{j}, \sigma_{j}\right)\right),
$$

where $x_{j}$ is the contribution of $j$ th mode of the vibration system, $\omega_{j}$ and $\sigma_{j}$ are the $j$ th mode frequency and damping factor, and $A$ and $\varphi$ are the amplitude and initial phase of $j$ th mode. This equation means that the response to any DOF can be represented by the decayed-attenuation sine function $e^{-\sigma_{j} t} \sin \left(\omega_{j} t+\varphi\left(\omega_{j}, \sigma_{j}\right)\right)$. Based on this, we set $x(n)$ as the discrete time series of an arbitrary DOF response in the vibrating system, $n=0,1, \ldots, N-1$, where $N$ is the length of time series. With interest frequency $\omega$ and damping factor

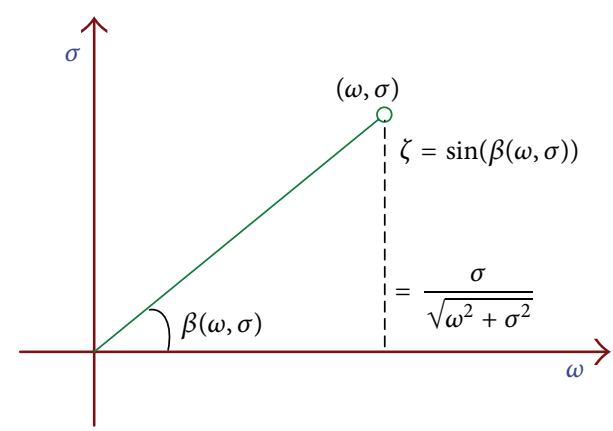

FIGURE 1: Schematic 2D representation of the vibration response.

$\sigma, x(n)$ can be written as the sum of a decayed-attenuation sine term modulated by the amplitude function $a(\omega, \sigma)$ and a residual term $w_{\omega, \sigma}(n)$, as follows:

$$
x(n)=a(\omega, \sigma) e^{-\sigma n} \sin (\omega n+\varphi(\omega, \sigma))+w_{\omega, \sigma}(n) .
$$

In (2), $a(\omega, \sigma)$ and $\varphi(\omega, \sigma)$ are the amplitude function and the phase function, respectively, of the point $(\omega, \sigma)$, which is defined in a $2 \mathrm{D}$ plane; $w_{\omega, \sigma}(n)$ includes the decayed attenuation sine terms of all points except for $(\omega, \sigma)$ and the random noise $w(n)$.

For the single DOF free response,

$$
\begin{gathered}
a(\omega, \sigma)=\sqrt{x(0)^{2}+\left(\frac{\dot{x}(0)^{2}-\sigma x(0)}{\omega}\right)}, \\
\varphi(\omega, \sigma)=\arctan \left(\frac{x(0) \omega}{\dot{x}(0)-\sigma x(0)}\right),
\end{gathered}
$$

where $x(0)$ and $\dot{x}(0)$ are the initial displacement and the initial velocity, respectively, when $t=0$ (or $n=0$ ).

We define the relationship between the damping factor $\sigma$ and the damping ratio $\zeta$ as follows:

$$
\zeta=\frac{\sigma}{\sqrt{\omega^{2}+\sigma^{2}}}=\frac{\sigma}{\sqrt{(2 \pi f)^{2}+\sigma^{2}}} .
$$

The geometrical meaning can be explained as the sine vector between the point $(\omega, \sigma)$ and the original point in a $2 \mathrm{D}$ plane, as shown in Figure 1.

2.2. Damped CAPES Spectral Estimation for Real-Valued Vibration Response Data. In (2), the time series $x(n)$ can be written as

$$
\begin{aligned}
x(n)= & a(\omega, \sigma) \cos (\phi(\omega, \sigma)) e^{-\sigma n} \sin (\omega n) \\
& +a(\omega, \sigma) \sin (\phi(\omega, \sigma)) e^{-\sigma n} \cos (\omega n) \\
& +w_{\omega, \sigma}(n) .
\end{aligned}
$$

If we let

$$
\begin{aligned}
& a_{c}(\omega, \sigma)=a(\omega, \sigma) \cos (\varphi(\omega, \sigma)) \\
& a_{s}(\omega, \sigma)=a(\omega, \sigma) \sin (\varphi(\omega, \sigma))
\end{aligned}
$$


then

$$
\begin{aligned}
x(n)= & a_{c}(\omega, \sigma) e^{-\sigma n} \cos (\omega n)+a_{s}(\omega, \sigma) e^{-\sigma n} \sin (\omega n) \\
& +w_{\omega, \sigma}(n) .
\end{aligned}
$$

(i) Hankel Matrix Method. We can obtain $N$ practical samples of the time series $x(n)$, such as $x(0), x(1), \ldots, x(N-1), n=$ $0,1, \ldots, N-1$. According to the nonparametric adaptive filter-bank spectral estimation method [1], we can construct the $M \times L$-order Hankel matrix of observation data $x(n)$,

$$
\begin{aligned}
\mathbf{Y} & =\left[\begin{array}{lllll}
\mathbf{y}_{0} & \cdots & \mathbf{y}_{L-1}
\end{array}\right] \\
& =\left[\begin{array}{ccccc}
x(0) & x(1) & \cdots & x(L-2) & x(L-1) \\
x(1) & x(2) & \cdots & x(L-1) & x(L) \\
\vdots & \vdots & \ddots & \vdots & \vdots \\
x(M-2) & x(M-1) & \cdots & x(N-3) & x(N-2) \\
x(M-1) & x(M) & \cdots & x(N-2) & x(N-1)
\end{array}\right] .
\end{aligned}
$$

In (8), $M$ is the user parameter, also known as the finite impulse response (FIR) filter length [9], $M \leq N / 2$, and $L=$ $N-M+1$ represents the total number of snapshots in the time series.

If we set

$$
\begin{aligned}
& \mathbf{A}_{M}(\omega, \sigma) \\
& =\left[\begin{array}{cc}
1 & 0 \\
e^{-\sigma} \cos (\omega) & e^{-\sigma} \sin (\omega) \\
\vdots & \vdots \\
e^{-\sigma(M-2)} \cdot \cos (\omega(M-2)) & e^{-\sigma(M-2)} \cdot \sin (\omega(M-2)) \\
e^{-\sigma(M-1)} \cdot \cos (\omega(M-1)) & e^{-\sigma(M-1)} \cdot \sin (\omega(M-1))
\end{array}\right], \\
& \Theta(\omega, \sigma)=\left[\begin{array}{cc}
a_{c}(\omega, \sigma) & a_{s}(\omega, \sigma) \\
a_{s}(\omega, \sigma) & -a_{c}(\omega, \sigma)
\end{array}\right] \\
& \mathbf{V}_{\omega, \sigma}(l)=\left[e^{-\sigma(l-1)} \cos (\omega(l-1)) e^{-\sigma(l-1)} \sin (\omega(l-1))\right] \\
& \mathbf{B}_{\omega, \sigma}(l)=\left[\begin{array}{cc}
e^{-\sigma(l-1)} \cos (\omega(l-1)) & e^{-\sigma(l-1)} \sin (\omega(l-1)) \\
-e^{-\sigma(l-1)} \sin (\omega(l-1)) & e^{-\sigma(l-1)} \cos (\omega(l-1))
\end{array}\right], \\
& \boldsymbol{\theta}(\omega, \sigma)=\left[\begin{array}{ll}
a_{c}(\omega, \sigma) & a_{s}(\omega, \sigma)
\end{array}\right]^{T}=\boldsymbol{\Theta} \cdot\left[\begin{array}{ll}
1 & 0
\end{array}\right]^{T} .
\end{aligned}
$$

Here, in (11) and (12), $l=0,1, \ldots, L-1$.

Then

$$
\begin{aligned}
\mathbf{y}_{l} & =\mathbf{A}_{M}(\omega, \sigma) \cdot \mathbf{B}_{\omega, \sigma}(l) \cdot \boldsymbol{\theta}(\omega, \sigma)+\mathbf{n}_{l} \\
& =\mathbf{H}_{\omega, \sigma}(l) \cdot \boldsymbol{\theta}(\omega, \sigma)+\mathbf{n}_{l} \\
& =\mathbf{A}_{M}(\omega, \sigma) \cdot \boldsymbol{\Theta}(\omega, \sigma) \cdot \mathbf{V}_{\omega, \sigma}(l)+\mathbf{n}_{l}
\end{aligned}
$$

and $\mathbf{H}_{\omega, \sigma}(l)=\mathbf{A}_{M}(\omega, \sigma) \cdot \mathbf{B}_{\omega, \sigma}(l)$. Equation (8) becomes

$$
\mathbf{Y}=\mathbf{A}_{M}(\omega, \sigma) \cdot \boldsymbol{\Theta}(\omega, \sigma) \cdot\left(\mathbf{A}_{L}(\omega, \sigma)\right)^{T}+\mathbf{n},
$$

where noise matrix is $\mathbf{n}$,

$$
\mathbf{n}=\left[\begin{array}{lll}
\mathbf{n}_{0} & \cdots & \mathbf{n}_{L-1}
\end{array}\right] .
$$

We now construct the weighted secondary residuals term J as the least squares estimator, as follows:

$$
\begin{aligned}
\mathbf{J}(\omega, \sigma)= & \left(\boldsymbol{\Phi}^{-1 / 2}(\omega, \sigma) \cdot \mathbf{W}(\omega, \sigma)\right)^{T} \\
& \times\left(\boldsymbol{\Phi}^{-1 / 2}(\omega, \sigma) \cdot \mathbf{W}(\omega, \sigma)\right),
\end{aligned}
$$

where $\Phi(\omega, \sigma)$ is the weight matrix and $\Phi^{-1 / 2}$ denotes the square root of Hermitian matrix $\boldsymbol{\Phi}$. W $(\omega, \sigma)$ is defined as

$$
\mathbf{W}(\omega, \sigma)=\mathbf{Y}-\mathbf{A}_{M}(\omega, \sigma) \cdot \boldsymbol{\Theta}(\omega, \sigma) \cdot\left(\mathbf{A}_{L}(\omega, \sigma)\right)^{T}
$$

Then, according to the nonparametric adaptive filterbank theory on spectral estimation, the weighted least squares estimator of parameter $\Theta$, which includes amplitude information, is given by

$$
\widehat{\boldsymbol{\Theta}}(\omega, \sigma)=\underset{\Theta(\omega, \sigma)}{\arg \min }\|\mathbf{J}(\omega, \sigma)\|^{2} .
$$

According to Larsson and Li's contribution [10], the final estimation of parameter $\boldsymbol{\theta}$ can be expressed as follows:

$$
\widehat{\boldsymbol{\theta}}(\omega, \sigma)=\frac{\mathbf{G}_{(\omega, \sigma)}^{-1} \cdot \mathbf{C}_{(\omega, \sigma)}^{T} \cdot \boldsymbol{\Phi}^{-1}(\omega, \sigma) \cdot \mathbf{A}_{M}(\omega, \sigma)}{\left(\mathbf{A}_{M}(\omega, \sigma)\right)^{T} \cdot \boldsymbol{\Phi}^{-1}(\omega, \sigma) \cdot \mathbf{A}_{M}(\omega, \sigma)} \cdot\left[\begin{array}{l}
1 \\
0
\end{array}\right]
$$

Here, differently with the Larsson and Li's expressions, $\mathbf{A}_{M}$ is defined as (9) and $\mathbf{G}_{(\omega, \sigma)}, \mathbf{C}_{(\omega, \sigma)}$ should be defined by

$$
\begin{gathered}
\mathbf{G}_{(\omega, \sigma)}=\frac{1}{L} \sum_{l=0}^{L-1}\left(\mathbf{V}_{\omega, \sigma}(l)\right)^{T} \mathbf{V}_{\omega, \sigma}(l) \\
\mathbf{C}_{(\omega, \sigma)}=\frac{1}{L} \sum_{l=0}^{L-1} \mathbf{y}_{l} \cdot \mathbf{V}_{\omega, \sigma}(l) .
\end{gathered}
$$

The weighting function values are as follows:

$$
\begin{gathered}
\boldsymbol{\Phi}_{\text {Capon }}=\widehat{\mathbf{R}}=\frac{1}{L} \mathbf{Y} \mathbf{Y}^{T}=\frac{1}{L} \sum_{l=0}^{L-1} \mathbf{y}_{l} \cdot \mathbf{y}_{l}^{T}, \\
\boldsymbol{\Phi}_{\text {Apes }}=\widehat{\mathbf{R}}-\mathbf{C}_{(\omega, \sigma)} \mathbf{G}_{(\omega, \sigma)}^{-1} \mathbf{C}_{(\omega, \sigma)}^{T} .
\end{gathered}
$$

(ii) Vectorization of Hankel Matrix. According to (8), we define 


$$
\begin{aligned}
& \mathbf{Y}_{\mathrm{vec}}=\operatorname{vec}\left(y_{l}\right)=\left[\begin{array}{llll}
y_{0} & y_{1} & \cdots & y_{L-1}
\end{array}\right]^{T} \\
& =\left[\begin{array}{llllllllll}
x(0) & \cdots & x(M-1) & x(1) & \cdots & x(M) & \cdots & x(L-1) & \cdots & x(N-1)
\end{array}\right]^{T} .
\end{aligned}
$$

The length of $\mathbf{Y}_{\mathrm{vec}}$ is $M \times L$.

Let

$\mathbf{H}_{\omega, \sigma}(l)$

$=\mathbf{A}_{M}(\omega, \sigma) \cdot \mathbf{B}_{\omega, \sigma}(l)$

$=\left[\begin{array}{cc}1 & 0 \\ e^{-\sigma} \cos (\omega) & e^{-\sigma} \sin (\omega) \\ \vdots & \vdots \\ e^{-\sigma(M-2)} \cdot \cos (\omega(M-2)) & e^{-\sigma(M-2)} \cdot \sin (\omega(M-2)) \\ e^{-\sigma(M-1)} \cdot \cos (\omega(M-1)) & e^{-\sigma(M-1)} \cdot \sin (\omega(M-1))\end{array}\right]$

$\cdot\left[\begin{array}{cc}e^{-\sigma(l-1)} \cos (\omega(l-1)) & e^{-\sigma(l-1)} \sin (\omega(l-1)) \\ -e^{-\sigma(l-1)} \sin (\omega(l-1)) & e^{-\sigma(l-1)} \cos (\omega(l-1))\end{array}\right]$.

Then, according to (7),

$$
\mathbf{Y}_{\mathrm{vec}}=\mathbf{H} \cdot \boldsymbol{\theta}(\omega, \sigma)+\mathbf{n} .
$$

In (27),

$$
\begin{gathered}
\mathbf{H}=\left[\begin{array}{c}
\mathbf{H}_{\omega, \sigma}(0) \\
\mathbf{H}_{\omega, \sigma}(1) \\
\vdots \\
\mathbf{H}_{\omega, \sigma}(L-1)
\end{array}\right], \quad \mathbf{n}=\left[\begin{array}{c}
n_{1} \\
n_{2} \\
\vdots \\
n_{L}
\end{array}\right], \\
\mathbf{n}_{l}=\left[\begin{array}{c}
w_{\omega, \sigma}(l) \\
w_{\omega, \sigma}(l+1) \\
\vdots \\
w_{\omega, \sigma}(l+M-1)
\end{array}\right]
\end{gathered}
$$

where $\mathbf{Y}_{\text {vec }} \in \mathbf{R}^{M L \times 1}$ is the observation vector, $\mathbf{H} \in \mathbf{R}^{M L \times 2}$ is the observation matrix, $\mathbf{n} \in \mathbf{R}^{M L \times 1}$, and $\mathbf{n}_{l} \in \mathbf{R}^{M \times 1}, M L=$ $M \times L$.

Spectral estimation for a signal at point $(\omega, \sigma)$ has become the problem of parameter estimation of $\boldsymbol{\theta}$, based on $\mathbf{Y}_{\mathrm{vec}}$, while minimizing the estimated noise. This is a typical linear estimation problem that can be solved by the following weighted least squares estimator $\mathbf{J}^{*}$ as seen in [5]:

$$
\begin{gathered}
\mathbf{J}^{*}=\left(\mathbf{Y}_{\mathrm{vec}}-\mathbf{H} \cdot \boldsymbol{\theta}\right)^{T} \boldsymbol{\Phi}^{-1}\left(\mathbf{Y}_{\mathrm{vec}}-\mathbf{H} \cdot \boldsymbol{\theta}\right), \\
\widehat{\boldsymbol{\theta}}(\omega, \sigma)=\underset{\theta(\omega, \sigma)}{\arg \min } \mathbf{J}^{*}(\omega, \sigma),
\end{gathered}
$$

where $\Phi \in \mathbf{R}^{M L \times M L}$ is the weighted values matrix. When $\mathbf{H}^{T} \boldsymbol{\Phi}^{-1} \mathbf{H}$ is nonsingular, the former equation is solved by

$$
\widehat{\boldsymbol{\theta}}(\omega, \sigma)=\left(\mathbf{H}^{T} \boldsymbol{\Phi}^{-1} \mathbf{H}\right)^{-1} \mathbf{H}^{T} \boldsymbol{\Phi}^{-1} \mathbf{Y}_{\mathrm{vec}}
$$

and then (21) reaches a minimum.
When the weighted matrix $\Phi$ is set to unit matrix, we can get a two-dimensional spectral estimation of the real signal in the least squares sense as follows:

$$
\widehat{\boldsymbol{\theta}}_{\mathrm{sl}}(\omega, \sigma)=\left(\mathbf{H}^{T} \mathbf{H}\right)^{-1} \mathbf{H}^{T} \mathbf{Y}_{\mathrm{vec}} \cdot
$$

According to the basic idea of Capon spectral estimation, $\Phi$ is set to the covariance matrix of data as follows:

$$
\boldsymbol{\Phi}_{\text {Capon }}=\widehat{\mathbf{R}}=\mathbf{Y}_{\mathrm{vec}} \cdot \mathbf{Y}_{\mathrm{vec}}^{T}
$$

However, according to the idea of APES spectral estimation, $\Phi$ is the covariance estimation of the residual $n$, given by

$$
\boldsymbol{\Phi}_{\mathrm{Apes}}=\mathbf{Q}_{v}=\widehat{\mathbf{R}}-\mathbf{H} \cdot \widehat{\boldsymbol{\theta}}_{\mathrm{sl}}(\omega, \sigma) \cdot \widehat{\boldsymbol{\theta}}_{\mathrm{sl}}^{T}(\omega, \sigma) \cdot \mathbf{H}^{T}
$$

Thus, we have five 2D damping spectral estimations of a real vibration signal, see in (23), (24), (31), (32), and (33).

\section{Algorithm Implementation}

Previous methods of estimation required the inverse of large weighted-matrices to be calculated at each $2 \mathrm{D}$ point $(\omega, \sigma)$. To reduce the computation time, we can utilize a matrix inversion formula to calculate the inverses in advance, leaving only a matrix multiplication in every iteration step.

Let $\mathbf{A}, \mathbf{B}, \mathbf{U}$, and $\mathbf{V}$ be arbitrary matrices; according to the matrix inversion formula, we have

$$
(\mathbf{A}-\mathbf{U B V})^{-1}=\mathbf{A}^{-1}-\mathbf{A}^{-1} \mathbf{U}\left(\mathbf{I}+\mathbf{B V A}^{-1} \mathbf{U}\right)^{-1} \mathbf{B V A}^{-1} .
$$

Corresponding to (24), let $\mathbf{A}$ be $\widehat{\mathbf{R}}$, let $\mathbf{B}$ be $\mathbf{G}_{(\omega, \sigma)}^{-1}$, let $\mathbf{U}$ be $\mathbf{C}_{(\omega, \sigma)}$, and let $\mathbf{V}$ be $\mathbf{C}_{(\omega, \sigma)}^{T}$; we have

$$
\begin{aligned}
\left(\mathbf{\Phi}_{\text {Apes }}\right)^{-1}= & \left(\widehat{\mathbf{R}}-\mathbf{C}_{(\omega, \sigma)} \mathbf{G}_{(\omega, \sigma)}^{-1} \mathbf{C}_{(\omega, \sigma)}^{T}\right)^{-1} \\
= & \widehat{\mathbf{R}}^{-1}-\widehat{\mathbf{R}}^{-1} \mathbf{C}_{(\omega, \sigma)}\left(\mathbf{I}+\mathbf{G}_{(\omega, \sigma)}^{-1} \mathbf{C}_{(\omega, \sigma)}^{T} \widehat{\mathbf{R}}^{-1} \mathbf{C}_{(\omega, \sigma)}\right) \\
& \times \mathbf{G}_{(\omega, \sigma)}^{-1} \mathbf{C}_{(\omega, \sigma)}^{T} \widehat{\mathbf{R}}^{-1}
\end{aligned}
$$

and corresponding to (33), let

$$
\widehat{\mathbf{R}}_{\theta, \mathrm{sl}}(\omega, \sigma)=\widehat{\boldsymbol{\theta}}_{\mathrm{sl}}(\omega, \sigma) \cdot \widehat{\boldsymbol{\theta}}_{\mathrm{sl}}^{T}(\omega, \sigma)
$$




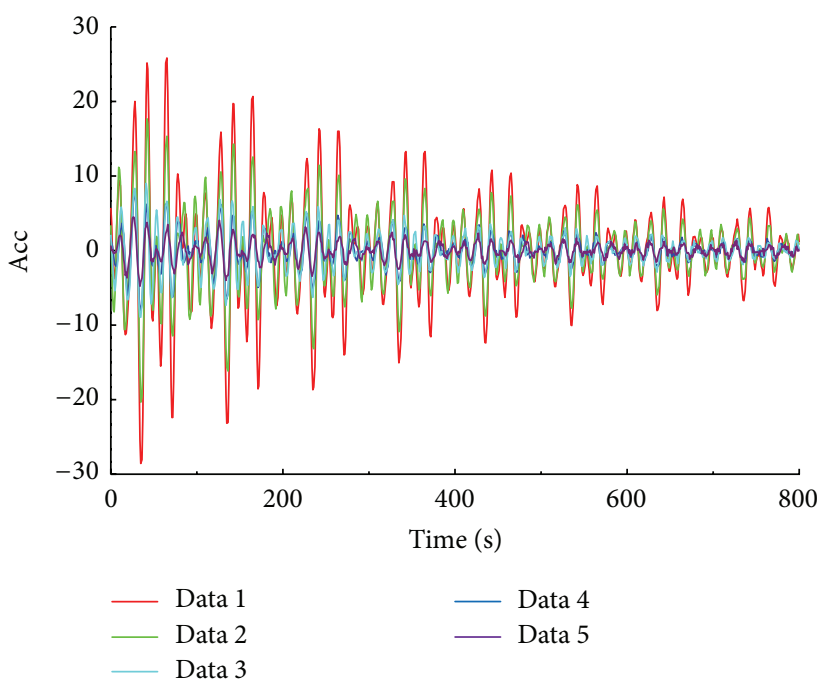

(a)

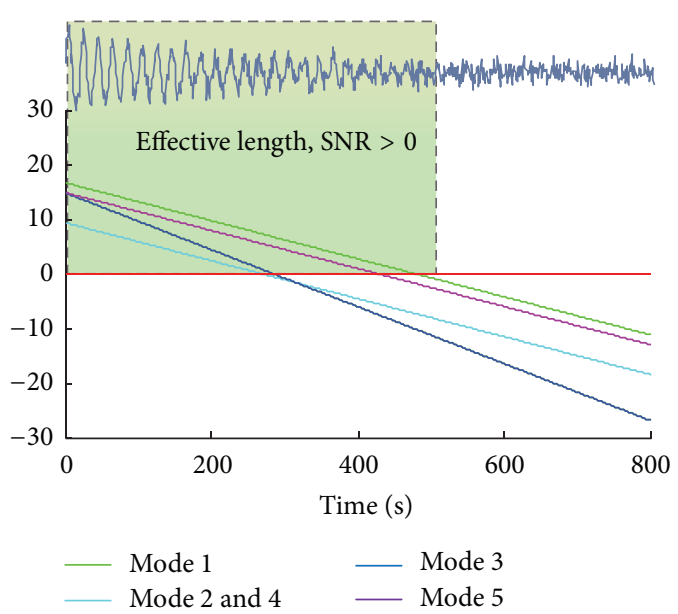

(b)

FIGURE 2: Free response history of a 5-DOF oscillation system: (a) data history; (b) effective length of each mode's contribution to the 1st DOF response.

This gives

$$
\begin{aligned}
\left(\boldsymbol{\Phi}_{\text {Apes }}\right)^{-1}= & \left(\widehat{\mathbf{R}}-\mathbf{H} \cdot \widehat{\mathbf{R}}_{\theta, \mathrm{sl}}(\omega, \sigma) \cdot \mathbf{H}^{T}\right)^{-1} \\
= & \left(\widehat{\mathbf{R}}-\mathbf{H} \cdot \widehat{\mathbf{R}}_{\theta, \mathrm{sl}} \cdot \mathbf{H}^{T}\right)^{-1} \\
= & \widehat{\mathbf{R}}^{-1}-\widehat{\mathbf{R}}^{-1} \cdot \mathbf{H} \\
& \cdot\left(\mathbf{I}+\widehat{\mathbf{R}}_{\theta, \mathrm{sl}} \cdot \mathbf{H}^{T} \cdot \widehat{\mathbf{R}}^{-1} \cdot \mathbf{H}\right) \widehat{\mathbf{R}}_{\theta, \mathrm{sl}} \cdot \mathbf{H}^{T} \cdot \widehat{\mathbf{R}}^{-1} .
\end{aligned}
$$

The large matrix inversions in (30) and (32) do not depend on the point $(\omega, \sigma)$; therefore, these can be calculated before entering the iteration cycle.

Hence, we design the proposed real damped version of CAPES (DR-CAPES) as follows:

step 1: set the data as $x$, and the user data length as $M$;

step 2: calculate $L=N-M+1$, form the $M \times L$-order Hankel matrix $\mathbf{R}$ and calculate $\mathbf{R}^{-1}$;

step 3: form the $2 \mathrm{D}$ grid $\left(\omega_{i}, \sigma_{j}\right)$, where $i$ and $j$ are the divisors of the frequency and damping factor, respectively; $i=$ $1,2, \ldots, i i, j=1,2, \ldots, j j$;

step 4: incrementing $i, j$ in a loop, calculate $\mathbf{G}_{(\omega, \sigma)}$ and $\mathbf{C}_{(\omega, \sigma)}$ according to (21) and (22); calculate $\left(\boldsymbol{\Phi}_{\text {Apes }}\right)^{-1}$ according to (35); calculate $\widehat{\boldsymbol{\theta}}_{\mathrm{wsl}}(\omega, \sigma)$ according to $(20)$; calculate the amplitude and phase of the real damping vibration signal according to (7).

\section{Numerical Case Studies}

Consider a 5-DOF linear oscillation system with the following structural matrix:

$$
\begin{gathered}
\mathbf{M}=\operatorname{diag}(1,2,1,4,8), \\
\mathbf{K}=\operatorname{diag}(0.0987,0.2843,0.1934,1.0107,2.5582), \\
\mathbf{C}=\operatorname{diag}(0.004,0.008,0.004,0.024,0.032) .
\end{gathered}
$$

It should be noted that, in order to simplify the problem and focus on the process of vibration signal itself, this numerical case of 5-DOF oscillation system is designated as full decoupling system, with all of its physical matrices $\mathbf{K}$, $\mathbf{M}$, and $\mathbf{C}$ being diagonal matrix. The modal parameters of the complex modal matrix, angular frequency, and damping factor can be calculated analytically as follows:

$$
\begin{gathered}
\boldsymbol{\omega}=2 \pi \cdot(0.05,0.06,0.07,0.08,0.09)^{T}, \\
\boldsymbol{\sigma}=(0.003,0.002,0.002,0.003 .0 .002)^{T}, \\
\langle\Psi|=\left[\begin{array}{ccccc}
12.3 & 10.0 & 5.3 & 10.0 & 10.0 \\
5.0 & 4.0 & 4.0 & 2.0 & 5.0 \\
4 & 7 & 3 & 7 & 2 \\
1 & 5 & 6 & 8 & 4 \\
7 & 4 & 7 & 3 & 1
\end{array}\right], \\
\hline \Psi=\left[\begin{array}{ccccc}
0.10 & 0.12 & 0.17 & 0.16 & 0.06 \\
0.01 & 0.08 & 0.05 & 0.11 & 0.19 \\
0.20 & 0.08 & 0.04 & 0.27 & 0.11 \\
0.21 & 0.26 & 0.17 & 0.38 & 0.26 \\
0.11 & 0.23 & 0.18 & 0.25 & 0.27
\end{array}\right] .
\end{gathered}
$$

The free vibration responses are generated from the initial status vector $\mathbf{x}_{0}=(2,3,4,5,1)^{T}$. These responses are 


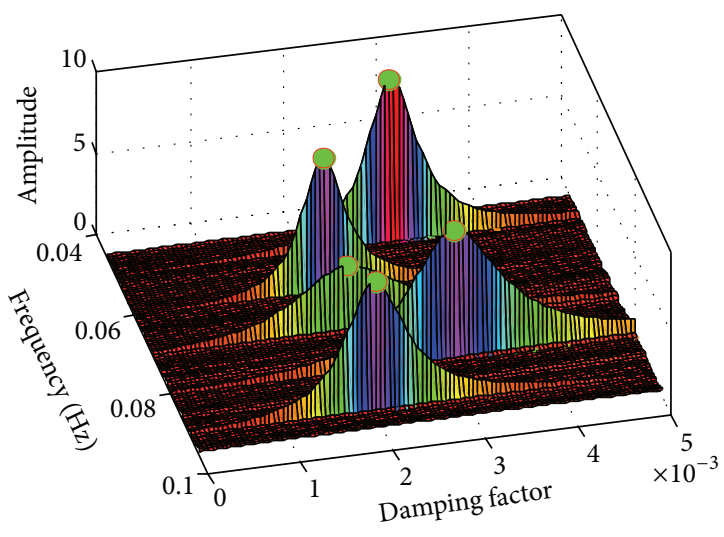

(a)

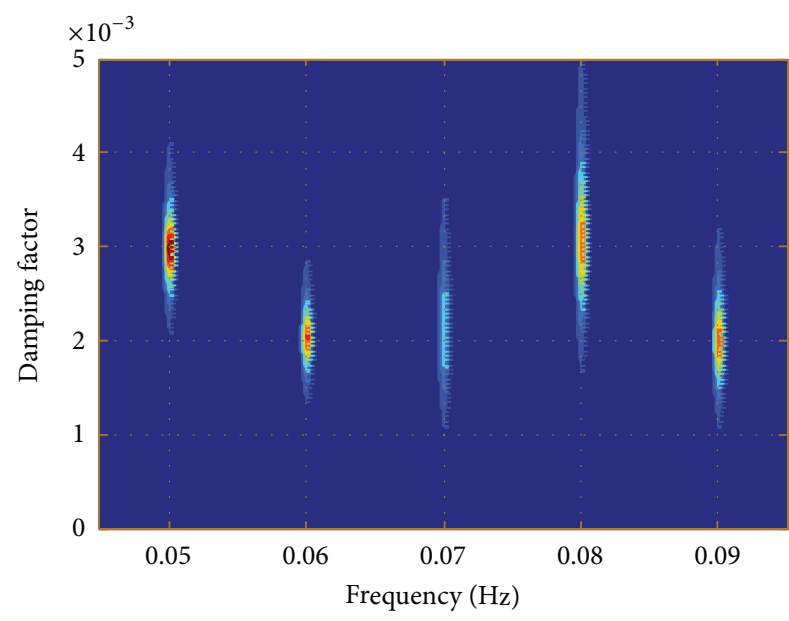

(b)

FiguRE 3: 2D amplitude spectrum estimated by the proposed approach (the response of the first DOF $x_{1}$ is chosen): (a) 2D amplitude of $x_{1}$; (b) contour of the amplitude spectrum in the $f, \sigma$ plane.

TABLE 1: Identified spectrum data.

\begin{tabular}{lccccc}
\hline Modes & Mode 1 & Mode 2 & Mode 3 & Mode 4 & Mode 5 \\
\hline Designated frequency & 0.05 & 0.06 & 0.07 & 0.08 & 0.09 \\
Identified frequency & 0.05 & 0.06 & 0.07 & 0.09 \\
Frequency error & 0 & 0 & 0 & 0 & 0.00 \\
Designated damping factor & 0.003 & 0.002 & 0.002 & 0.003 & 0.002 \\
Identified damping factor & 0.00301 & 0.00213 & 0.00215 & 0.002 \\
Damping factor error (\%) & 0.33 & 6.5 & 7.5 & 0.67 \\
\hline
\end{tabular}

observed fully under an initial signal-to-noise ratio (SNR) of

$$
\mathbf{R}_{\mathrm{SN}, 0}=\left[\begin{array}{ccccc}
16.6 & 14.8 & 9.3 & 14.8 & 14.8 \\
13.1 & 11.1 & 11.1 & 5.1 & 13.1 \\
2.0 & 6.8 & -0.5 & 6.9 & -4.0 \\
-8.6 & 5.4 & 7.0 & 9.5 & 3.5 \\
1.9 & -3.0 & 1.9 & -5.4 & -15
\end{array}\right]
$$

and a sampling frequency of $1.0 \mathrm{~Hz}$, as shown in Figure 2(a). The total length of the data is 800 samples and there are 40 , $48,56,64$, and 72 characteristic periods for each mode. If we define the effective length of the data to be the segment with a nonnegative SNR, these numbers are reduced to 24, 17, 21, 25, and 38, as illustrated in Figure 2(b). These characteristic periods are shorter than any other spectral estimation approaches, thereby illustrating the effectiveness of feature extraction in the proposed approach.

The response of the first DOF $x_{1}$ has the biggest initial SNR of all the responses. Therefore, $x_{1}$ was chosen to feed the proposed approach. The 3D amplitude spectrum and its corresponding contour in the $(f, \sigma)$-plane are shown in Figure 3.

In Figure 3(a), the five distinct peaks lie in the plane of the natural frequency and damping factor, that is, $f, \sigma$, respectively. The bases of the peaks are well separated from each other and the widths in the frequency dimension are so narrow that each peak looks like a thin blade. In the damping factor dimension, the slopes of the ridges are gentle and the bases are wide. Each apex is located exactly at its corresponding designated value, as shown in Figure 3(b). The identified spectrum data are listed in Table 1.

The proposed approach performed well in identifying both the frequency and damping factor. Under the noise levels given above, the frequency errors were almost zero. The damping factor errors were also smaller with the proposed method than with any other approach.

4.1. Frequency Resolution of 3D Amplitude Spectrum. The traditional CAPES method is known for its high frequency resolution and accurate spectral value estimation. To investigate the frequency resolution of the proposed DR-CAPES approach, we conducted a case study based on the oscillation system described above.

The effect of closed peaks was simulated by changing the physical parameters $\mathbf{K}, \mathbf{M}$, or $\mathbf{C}$. In this case, we simply fixed the second mode to $0.06 \mathrm{~Hz}$ and gradually adjusted the first mode frequency from $0.05 \mathrm{~Hz}$ to $0.06 \mathrm{~Hz}$ while maintaining $\mathbf{M}$ and C. A new 5-DOF oscillation system with a different $\mathbf{K}$ was obtained for each iteration and the free vibration responses of the system were calculated under the initial SNRs mentioned above.

Figure 4 shows the amplitude spectra of four typical steps when the first mode approaches the second. The other modes were not identified and are not illustrated in the figures. As a comparison, the global amplitude spectrum identified by 


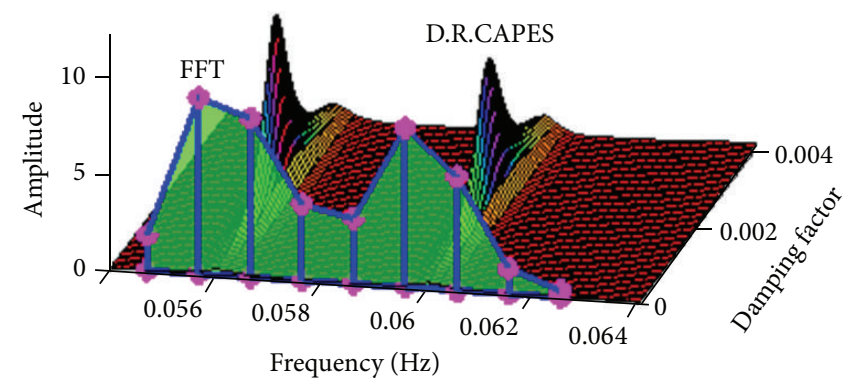

(a)

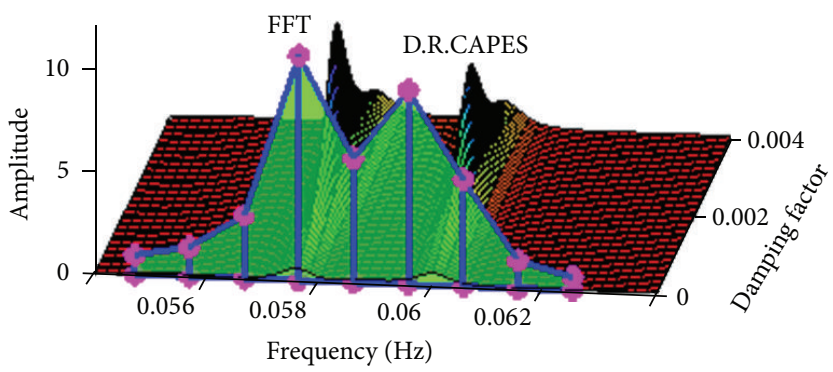

(c)

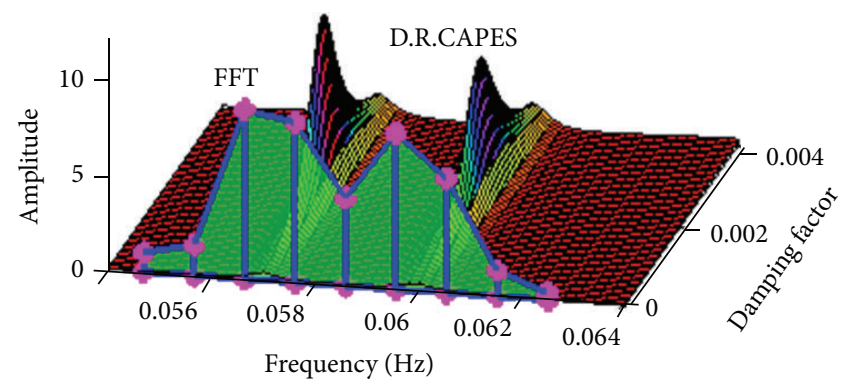

(b)

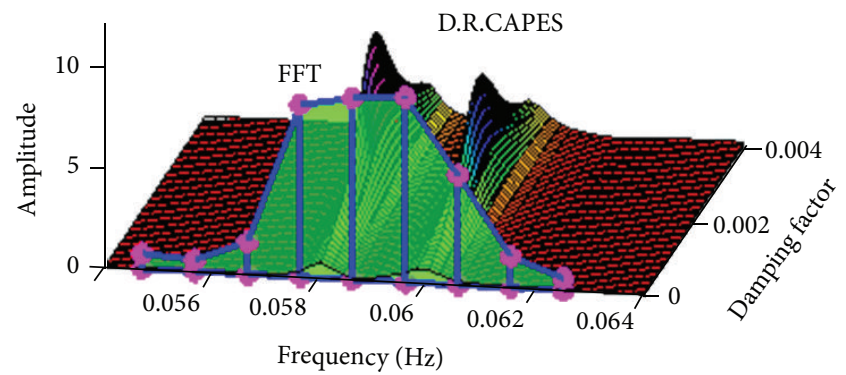

(d)

FIGURE 4: Comparison of frequency resolution of the amplitude spectrum using DR-CAPES and FFT. ((a) $f_{1}=0.052, f_{2}=0.06$; (b) $f_{1}=$ $0.054, f_{2}=0.06$; (c) $f_{1}=0.056, f_{2}=0.06$; (d) $f_{1}=0.058, f_{2}=0.06$; FFT spectrum in each figure was formed from data with a length of 1024 and a zero appending technique was used).

an FFT method was also found and the nine spectral lines corresponding to the same frequency window are plotted in the figures. It can be seen that the frequency resolution of DR-CAPES is much better than that of the FFT method; from Figures 4(a)-4(c), the two peaks of the FFT spectrum approach one another quickly and in Figure 4(d) the peaks have overlapped completely, whereas the two 3D peaks given by the DR-CAPES method remain separate and distinct, with the shape and height of the peaks basically invariant.

To further illustrate the high resolution of the proposed approach, we define a mode closeness measure and frequency resolution in a physical sense as follows.

Let

$$
\kappa=\log _{10} \delta
$$

be a measure of the closeness of two adjacent modes (or adjacent peaks) in a spectrum, in which

$$
\delta=\frac{\omega_{j+1}+\omega_{j}}{\omega_{j+1}-\omega_{j}}
$$

and $\omega_{j}$ is the $j$ th mode or peak in spectrum. Larger $\kappa$ values imply closer distances between two spectrum lines, modes, or peaks.

When adjacent peaks are close to each other, the physicalsense frequency resolution can be defined as the logarithm of a ratio of the minimum height of two adjacent peaks and the saddle value between them, as follows:

$$
\begin{gathered}
R_{\omega}=\log _{10} \alpha, \\
\alpha=\frac{\min \left(A_{1}, A_{2}\right)}{\min _{\omega_{1} \leq \omega \leq \omega_{2}}\left(A_{\omega}\right)} .
\end{gathered}
$$

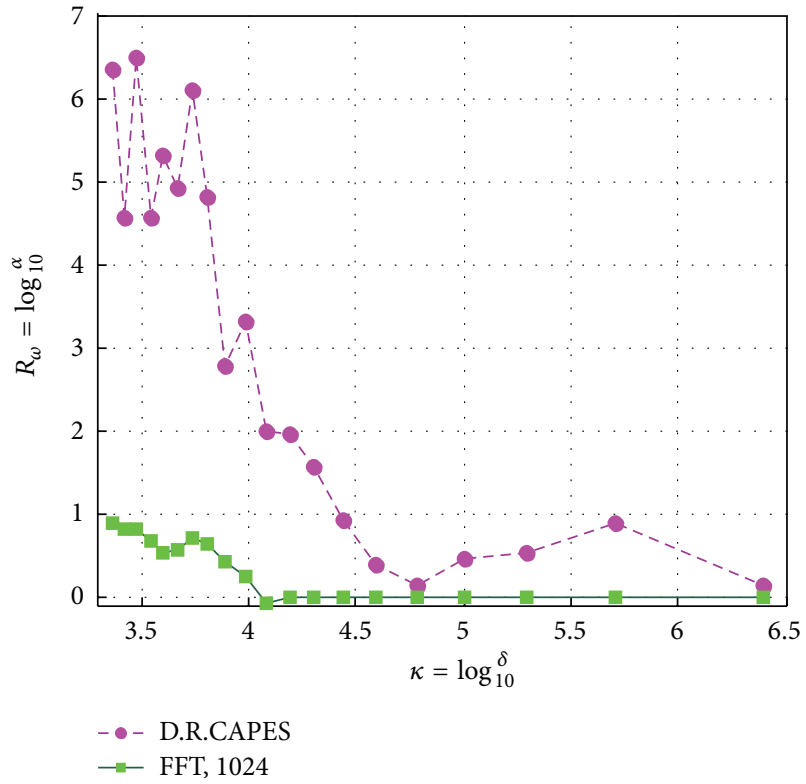

FIGURE 5: Comparison of frequency resolution between DR-CAPES method and FFT method.

Here, $A_{\omega}$ is the saddle value of two adjacent hills in the spectrum and $A_{1}, A_{2}$ are the values of the two adjacent peaks.

Figure 5 illustrates how the results of the two processes converge in terms of the mode closeness and frequency resolution. As the mode closeness increases, the frequency resolutions of both the DR-CAPES method and the FFT method decrease. The resolution of the FFT method dropped 


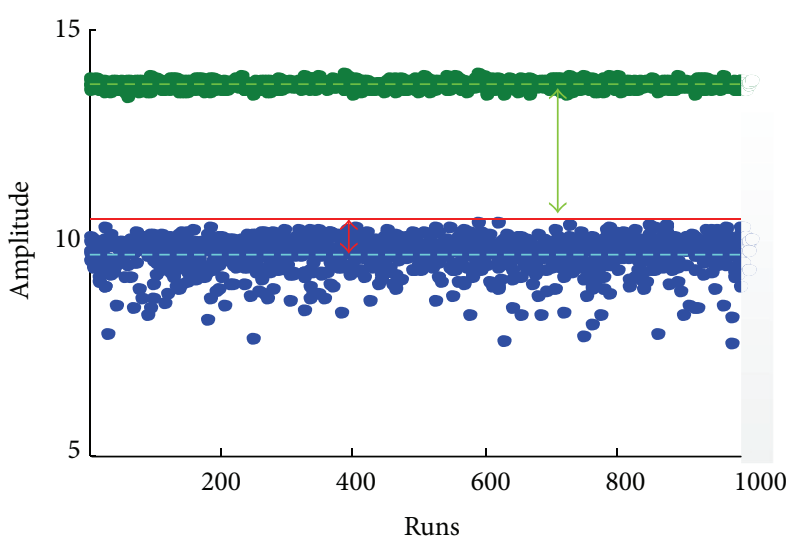

(a)

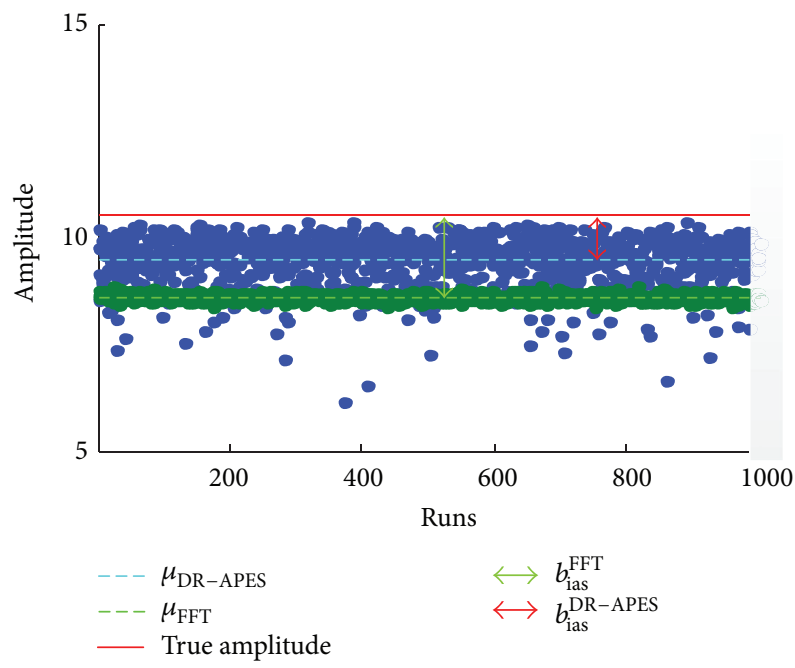

(c)

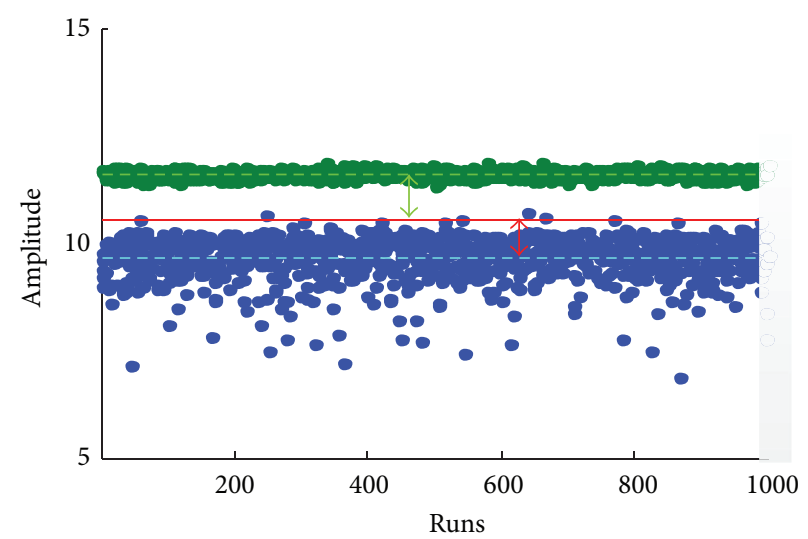

(b)

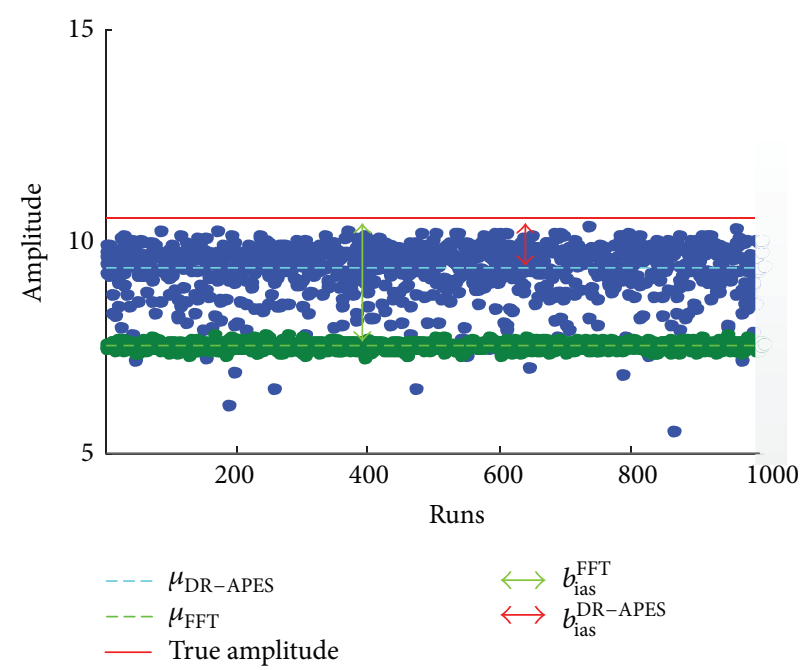

(d)

FIGURE 6: Scatterplot of the peak amplitude spectra given by DR-CAPES and FFT ((a) $\sigma=0.001$; (b) $\sigma=0.0015$; (c) $\sigma=0.0025$; (d) $\sigma=0.003)$.

close to zero at a relatively small value of mode closeness. Compared to FFT, the proposed DR-CAPES has a higher frequency resolution, even when the two peaks are extremely close to each other.

4.2. Bias and Accuracy of the Amplitude Spectrum Given by the DR-CAPES Method. The bias and accuracy of the amplitude spectrum identified by the DR-CAPES method can be revealed by numerical simulations. Four simulations were performed under a fixed observation noise level; that is, the initial SNR was $16.6 \mathrm{~dB}$ for the first mode component of the response corresponding to the 1st DOF. Each simulation was run with the same parameters, except for the damping factor, which was set as $\sigma=0.001,0.0015,0.0025$, and 0.003 . In each simulation, the following processes were repeated 1000 times: generation of random response, simultaneous spectrum estimation with the proposed method, and the FFT method.

Figure 6 shows the scatterplots of the simulation results. Each subplot corresponds to a designated damping factor, that is, $\sigma=0.001,0.0015,0.0025$, and 0.003 . The mean and bias of these estimates by these two methods, indicated as $\mu_{\mathrm{DR}-\mathrm{APES}}, \mu_{\mathrm{FFT}}, b_{\mathrm{ias}}^{\mathrm{FFT}}$, and $b_{\text {ias }}^{\mathrm{DR}-\mathrm{APES}}$, and the true values of the amplitude (designated value) are also plotted.

The results show that the spectrum bias of the DR-CAPES method is smaller than that of the FFT method. Regardless of the designated damping factor, the bias of the DR-CAPES method stabilized to a certain level below the true amplitude, whereas the FFT estimation was biased on either side depending on the designated damping factor. Additionally, as shown in Figure 6, the extent of scatter of the FFT result is smaller than that of the proposed method; this may mean that the accuracy of the proposed DR-CAPES method can be further improved.

4.3. Influence of Noise on the Amplitude Spectrum. In the above discussion, the noise is fixed at a very low level. Further examination investigated the influence of noise level on the quality of the estimated amplitude spectrum. Similar to the numerical simulation case studies above, we simulated 


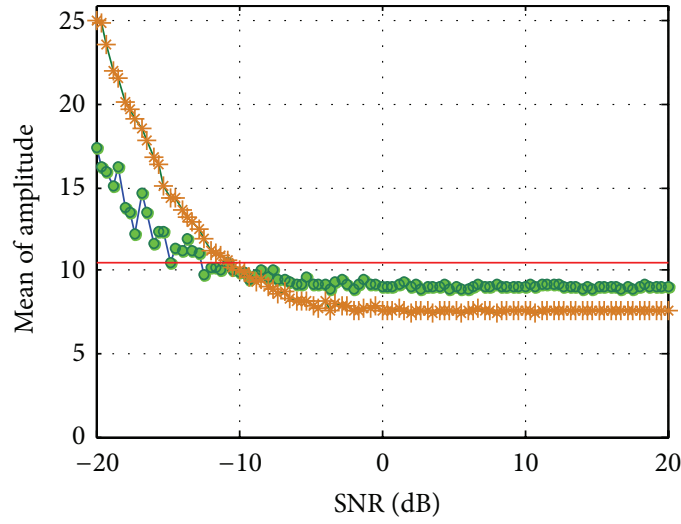

(a) $f=0.05 \mathrm{HZ}, \sigma=0.003$

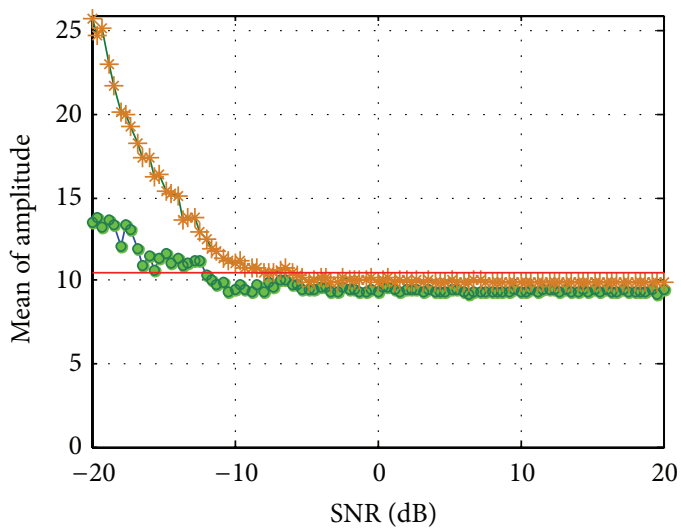

(c) $f=0.05 \mathrm{HZ}, \sigma=0.002$

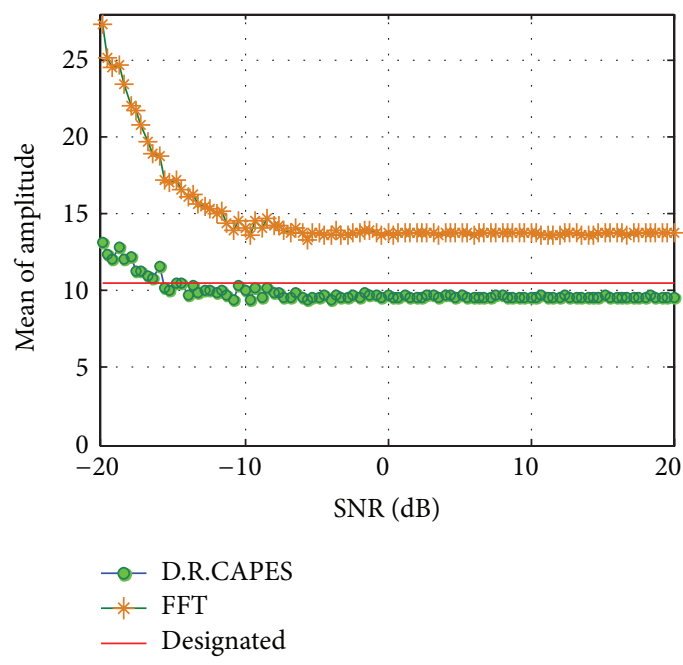

(e) $f=0.05 \mathrm{HZ}, \sigma=0.001$

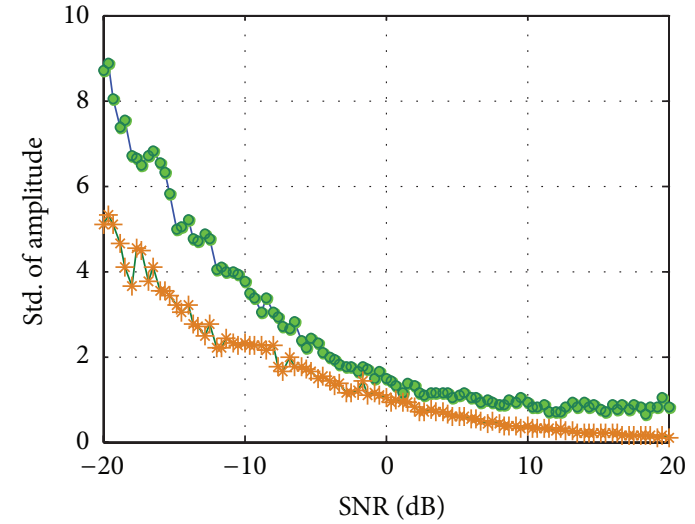

(b) $f=0.05 \mathrm{HZ}, \sigma=0.003$

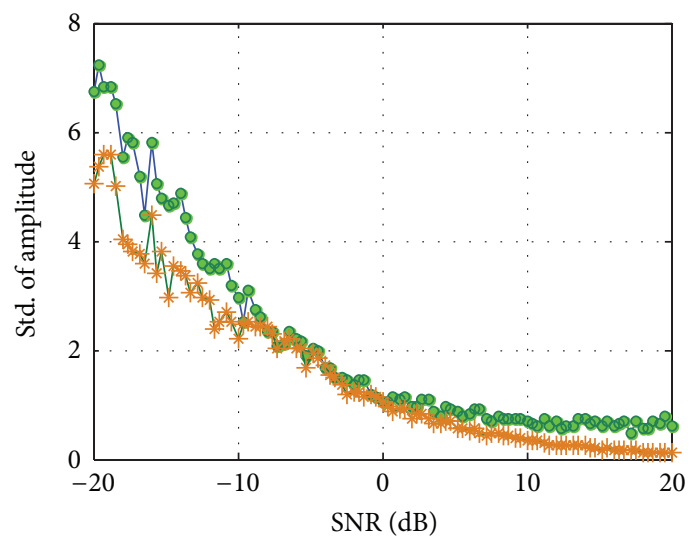

(d) $f=0.05 \mathrm{HZ}, \sigma=0.002$

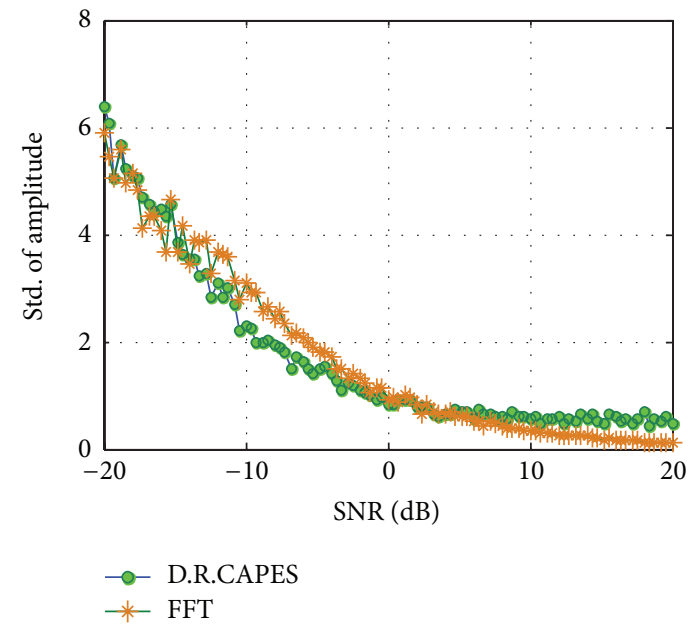

(f) $f=0.05 \mathrm{HZ}, \sigma=0.001$

FiguRE 7: Influence of noise on the identified amplitude spectrum, that is, mean and standard deviation. ((a), (b) mode 1, $\sigma=0.003$; (c), (d) mode $2, \sigma=0.002$; (e), (f) mode $3, \sigma=0.001$ ).

four different damping factors. In each simulation, the noise level was divided into 100 steps with an initial SNR interval of $[-20,20] \mathrm{dB}$. At each noise level, the simulation process included the generation of a random response and estimation of the amplitude spectrum using the proposed method and the FFT method. The mean and standard deviation of the results given by both methods at different SNRs are illustrated in Figure 7. For brevity, we only present three of the typical damping factors in this paper; that is, $0.003,0.002$, and 0.001 .

Figures $7(\mathrm{a}), 7(\mathrm{c})$, and $7(\mathrm{e})$ show that the proposed DRCAPES method produces less bias than the FFT method and that damping factor effects were separated from the 
amplitude effects in the amplitude spectrum estimation. The proposed method is not affected by the damping factor, whereas the FFT method performed poorly with a swingintolerant bias when the damping factor was changed.

The curves for both methods gently plateau when the initial SNR is set to a specific value, that is, $-6.0 \mathrm{~dB}$ for Figure 7(a) and $-10 \mathrm{~dB}$ for both Figures 7(c) and 7(e). Before this plateau, the mean of the estimated amplitude dropped sharply from a value that is far higher than the true amplitude or designated value. The plateau in the curves proves that an optimum workable SNR zone exists for each individual freevibration response, which implies that a denoising process is needed if the initial SNR of the raw response data is below this workable zone.

Figures $7(\mathrm{~b}), 7(\mathrm{~d})$, and $7(\mathrm{f})$ show that the observation noise level has a strong influence on the statistical qualities of the amplitude spectrum estimation, that is, the standard deviation $\sigma$. As the initial SNR was increased, the standard deviation of both methods decreased. Under most noise levels, the standard deviation of the proposed method was significantly greater than that of the FFT method. The reason for this may be that the spectrum estimation of the proposed method is more complicated than that of the FFT method, where variance is propagated. A higher SNR means a smaller standard deviation and statistically better spectrum estimation; this conclusion indicates that we should focus on improving the quality of the amplitude spectrum.

\section{Conclusion}

The vibration response of structures in the real physical world is evaluated and modulated by amplitude damping. By representing the free vibration response in two dimensions (frequency and damping factor), we extended the hybrid CAPES approach, which is a high-resolution amplitude spectrum estimation method, to estimate the amplitude spectrum of the structural vibration response. Compared to the FFT method, the proposed DR-CAPES has a much better resolution in the frequency domain and is, therefore, more suitable for overcoming the difficulties in spectrum estimation when vibration modes are close together. The downward bias of the amplitude spectrum estimated by DR-CAPES is smaller than that given by the FFT method and is not influenced by the damping factor; therefore, the proposed method is more accurate than FFT-like approaches when determining amplitude. However, with higher SNRs, secondary operations, such as averaging and denoising, are needed to improve the amplitude spectrum estimate.

\section{Notations}

\footnotetext{
$\sigma:$ Damping factor, decay

$\zeta$ : Damping ratio

$\delta$ : Logarithmic decrement

$c$ : Damping coefficient

$\omega_{d}$ : Damped natural angular frequency

$\omega_{n}$ : Natural angular frequency

$m_{j}$ : Lumped mass at the $j$ th degree of freedom (modal)
}

$k_{j}: \quad$ Modal stiffness at the $j$ th degree of free-

$x(0): \quad$ Initial displacement

$L: \quad$ Total number of snapshots in the time series

$\boldsymbol{\Phi}_{\text {Capon }}$ : Weight matrix for Capon spectral estimation method

$\mathbf{Y}_{\mathrm{vec}}$ : $\quad$ Vectorization of Hankel matrix

$\boldsymbol{\omega}$ : $\quad$ Angular frequency vector

$\angle \Psi: \quad$ Modal phase matrix

$\alpha: \quad$ Frequency resolution

$\mathbf{A}_{M}: \quad$ Defined as (9)

$R_{\omega}: \quad$ Logarithm of frequency resolution

$a_{s}$ : $\quad$ Sinusoidal component of amplitude

$c_{j}$ : Modal stiffness at the $j$ th degree of free-

m: Diagnosis mass matrix (lumped mass matrix)

k: $\quad$ Modal stiffness matrix (diagnosis)

c: Modal damping matrix (diagnosis)

M: $\quad$ Mass matrix

C: $\quad$ Damping matrix

K: $\quad$ Stiffness matrix

$\omega: \quad$ Angular frequency (measured)

$\dot{x}(0): \quad$ Initial velocity

Y: $\quad$ Hankel matrix of observation data

J: Least squares estimator matrix of the weighted secondary residuals term $J$

$\Phi_{\text {Apes }}$ : Weight matrix for APES spectral estimation method

H: Observation matrix

$\sigma: \quad$ Damping factor vector

$\mathbf{R}_{\mathrm{SN}, 0}$ : Initial signal-to-noise ratio of each contribution in each response

$\mathbf{G}_{(\omega, \sigma)}: \quad$ Defined by $(21)$

$\mathbf{B}_{\omega, \sigma}: \quad$ Defined by (12)

$a_{c}: \quad$ Cosine component of amplitude

$e: \quad$ Exponential function

$n$ : $\quad$ Number of degrees of freedom

$x: \quad$ Response of steady forced vibration

$A(\cdot), a(\cdot)$ : Amplitude function

$\varphi(\cdot)$ : $\quad$ Phase function

$t: \quad$ Time series

$f: \quad$ Natural frequency

$w: \quad$ Residual term

$N$ : $\quad$ Length of the time series

M: $\quad$ Finite impulse response (FIR) filter length

$\boldsymbol{\theta}, \widehat{\boldsymbol{\theta}}_{\mathrm{wsl}}$ : Solution to the weighted least squares parameter and its estimator

$\boldsymbol{\Theta}, \widehat{\Theta}: \quad$ Weighted least squares parameter and its estimator

$\widehat{\boldsymbol{\theta}}_{\mathrm{sl}}$ : $\quad$ 2D spectral estimation of real signal in the sense of least squares

$|\Psi|$ : $\quad$ Modal amplitude matrix

$\kappa$ : Closeness of two adjacent modes (or adjacent peaks) in spectrum 
$\mathrm{C}_{(\omega, \sigma)}$ : Defined by (22)

$\mathbf{V}_{\omega, \sigma}$ : Defined by (11)

n: $\quad$ Noise matrix.

\section{Conflict of Interests}

The authors declare that there is no conflict of interests regarding the publication of this paper.

\section{Acknowledgments}

This research is supported by the State High-Tech Research and Development Plans (863), Grant no. 2014AA110402; State Meteorological Administration Special Funds of Meteorological Industry Research, Grant no. 201306102; the Project of National Key Technology R\&D Program in the 12th Five Year Plan of China (Grant no. 2012BAJ11B01); the Fundamental Research Funds for the Central Universities.

\section{References}

[1] J. Penzien, "Dynamics of structures," Computers and Structures, Inc., 2003.

[2] D. J. Ewins, "Modal Testing, Theory, Practice, and Application," 2000.

[3] B. P. Lathi, Linear Systems and Signals, Oxford University Press, New York, NY, USA, 2nd edition, 2004.

[4] P. Stoica and R. Moses, Introduction to Spectral Analysis, Prentice Hall, Upper Saddle River, NJ, USA, 1997.

[5] D. P. Kalogeras, An Overview of the Spectral Estimation Methods, vol. 12 of Mathematics and Computers in Science and Engineering Series, 2013.

[6] D. G. Manolakis, V. K. Ingle, and S. M. Kogon, Statistical and Adaptive Signal Processing, McGraw-Hill, 2000.

[7] J. Capon and S. Haykin, "Maximum-likelihood spectral estimation," in Nonlinear Methods of Spectral Analysis, Springer, New York, NY, USA, 1983.

[8] J. Li and P. Stoica, "An adaptive filtering approach to spectral estimation and SAR imaging," IEEE Transactions on Signal Processing, vol. 44, no. 6, pp. 1469-1484, 1996.

[9] P. Stoica, H. Li, and J. Li, "New derivation of the APES filter," IEEE Signal Processing Letters, vol. 6, no. 8, pp. 205-206, 1999.

[10] E. G. Larsson and J. Li, High-Resolution Nonparametric Spectral Analysis: Theory and Applications, 2003.

[11] H. Li, J. Li, and P. Stoica, "Performance analysis of forwardbackward matched-filterbank spectral estimators," IEEE Transactions on Signal Processing, vol. 46, no. 7, pp. 1954-1966, 1998.

[12] A. Jakobsson and P. Stoica, "Combining Capon and APES for estimation of spectral lines," Circuits, Systems, and Signal Processing, vol. 19, no. 2, pp. 159-169, 2000.

[13] E. G. Larsson, G. Liu, P. Stoica, and J. Li, "High-resolution SAR imaging with angular diversity," IEEE Transactions on Aerospace and Electronic Systems, vol. 37, no. 4, pp. 1359-1372, 2001.

[14] P. Stoica and T. Sundin, "Nonparametric NMR spectroscopy," Journal of Magnetic Resonance, vol. 152, no. 1, pp. 57-69, 2001.

[15] Z.-S. Liu, R. Wu, and J. Li, "Complex ISAR imaging of maneuvering targets via the Capon estimator," IEEE Transactions on Signal Processing, vol. 47, no. 5, pp. 1262-1271, 1999.
[16] A. Jakobsson, T. Ekman, and P. Stoica, "CAPON and APES spectrum estimation for real-valued signals," in Proceedings of the IEEE Digital Signal Processing Workshop, August 1998.

[17] K. S. Ryu, M. Sepehrifar, and A. A. Kishk, "High accuracy peak location and amplitude spectral estimation via tuning APES method," Digital Signal Processing, vol. 20, no. 2, pp. 552-560, 2010.

[18] G. O. Glentis and A. Jakobsson, "Computationally efficient damped Capon and APES spectral estimation," in Proceedings of the 21st European Signal Processing Conference (EUSIPCO '13), 2013. 

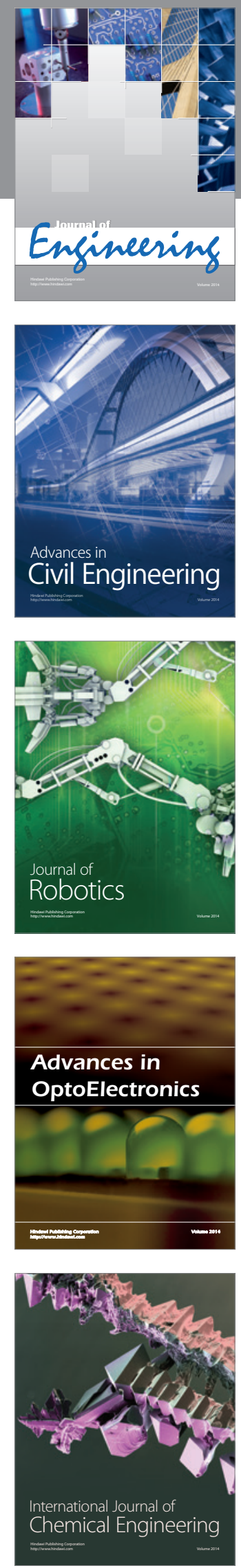

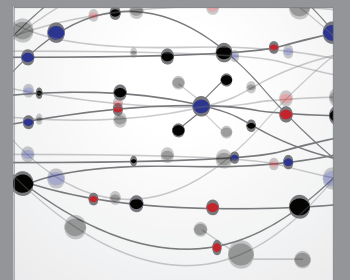

The Scientific World Journal
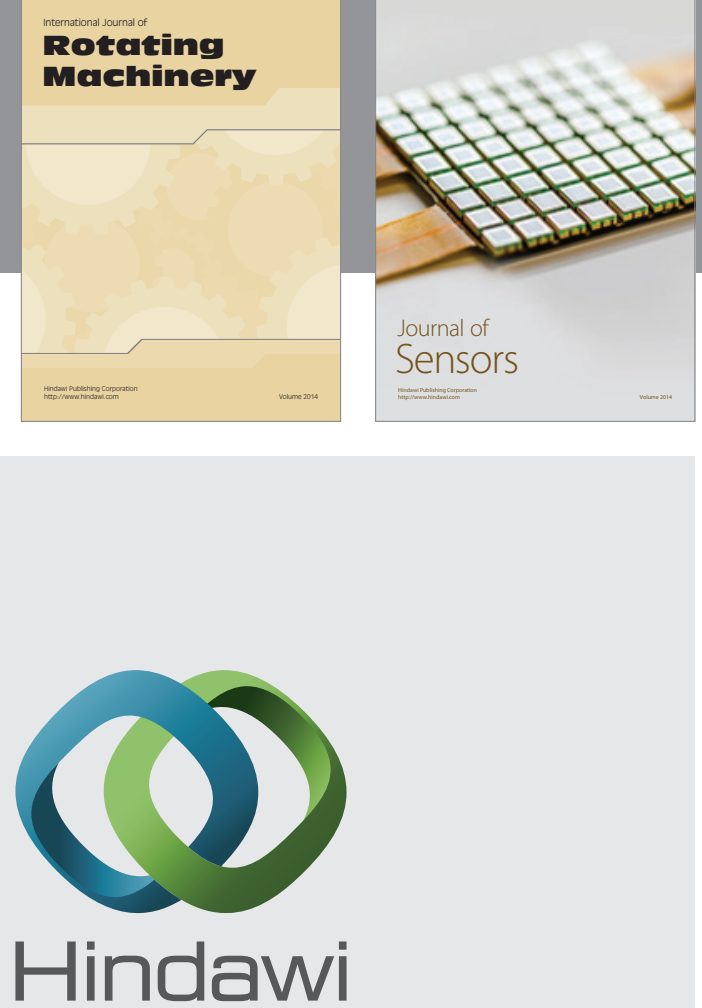

Submit your manuscripts at http://www.hindawi.com
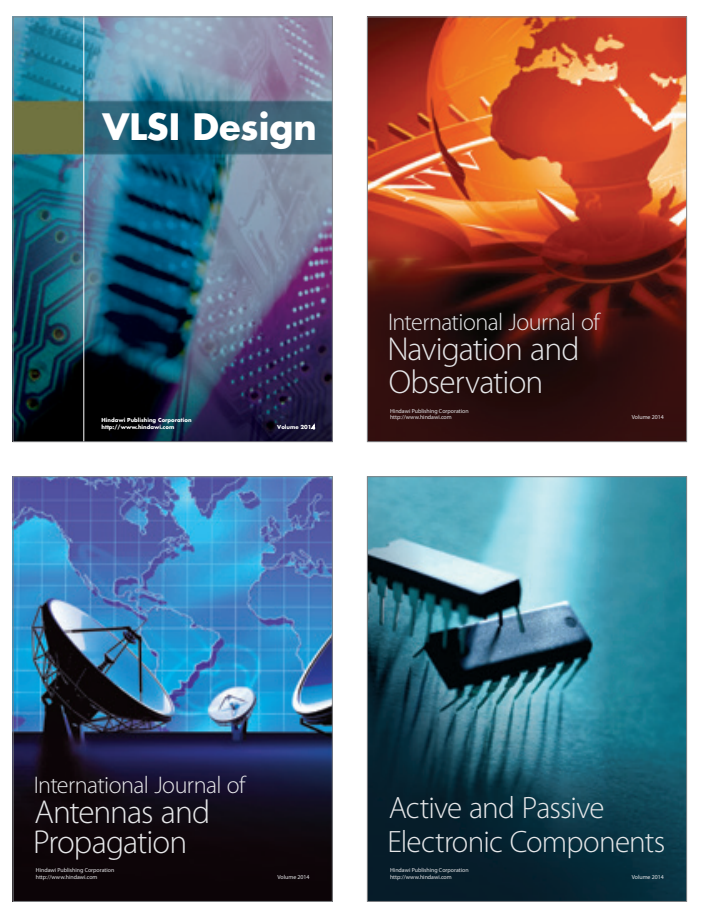
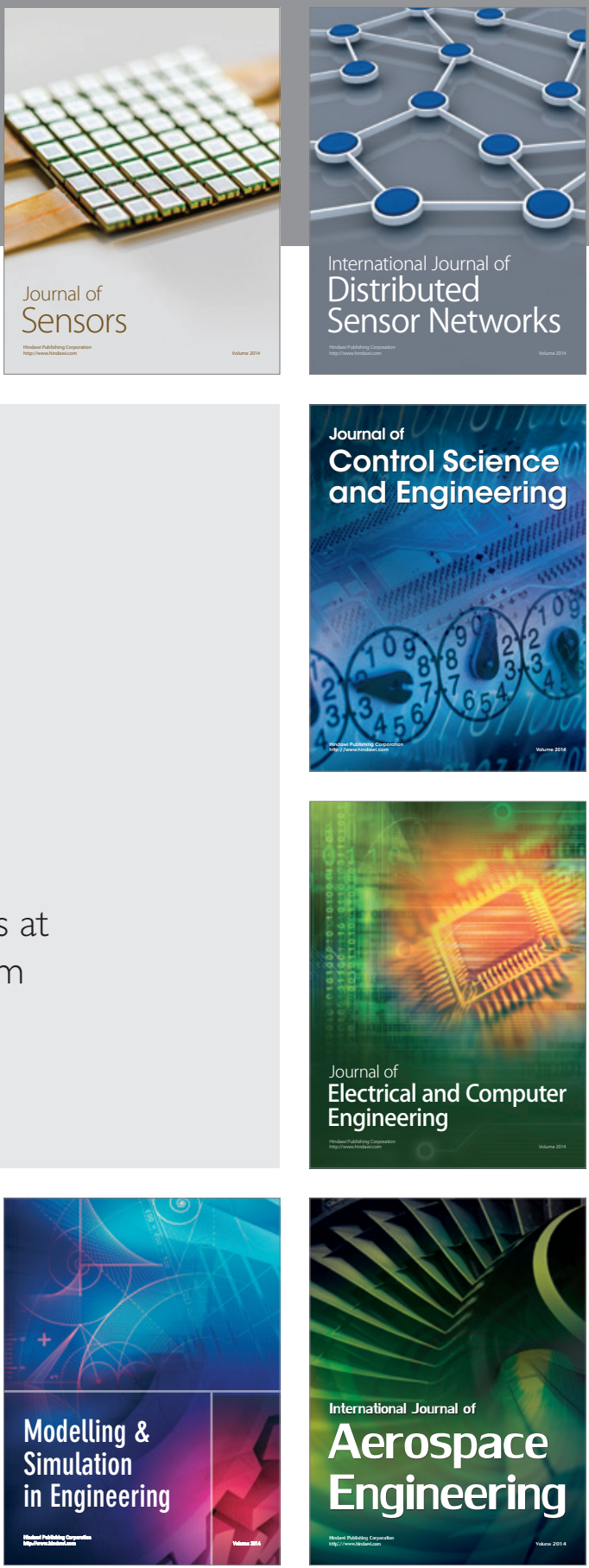

Journal of

Control Science

and Engineering
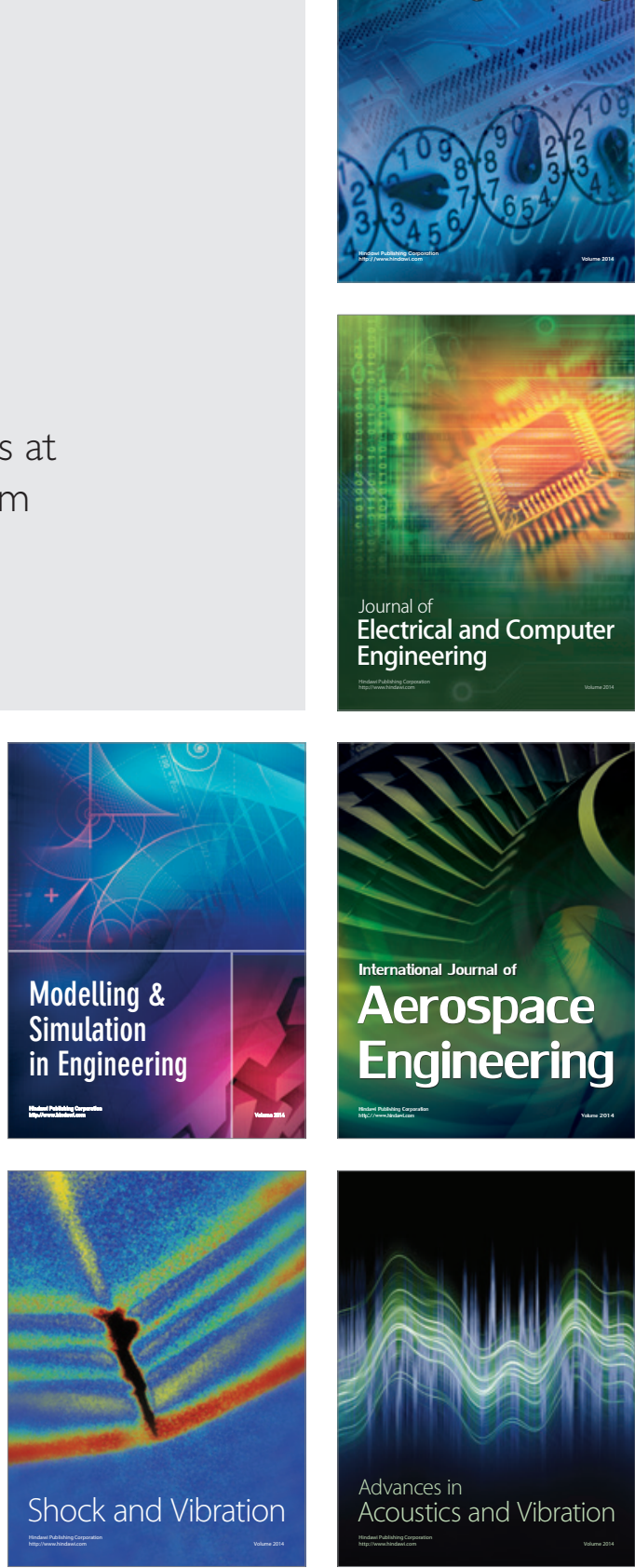